\title{
Brain Tumour Classification using Deep Learning with Residual Attention Network: A Comparative Study
}

\author{
Abdulrazak Yahya Saleh \\ FSKPM Faculty \\ Universiti Malaysia Sarawak (UNIMAS) \\ Kota Samarahan, Sarawak, Malaysia \\ ysahabdulrazak@unimas.my
}

\author{
Sashwini A/P S Thiagaraju \\ FSKPM Faculty \\ Universiti Malaysia Sarawak (UNIMAS) \\ Kota Samarahan, Sarawak, Malaysia \\ sashuwini1997@gmail.com
}

\begin{abstract}
The main goal of this paper is to evaluate the performance of deep learning with Residual Attention Network (RAN) for brain tumour classification. Digitalised Magnetic Resonance Image (MRI) datasets obtained from Malaysian hospitals and other sources are utilised in this paper. The MRI datasets consist of information of those patients who are 20 years old and above, both male and female. The RAN algorithm is trained and tested using the MRI datasets. The algorithm performance is evaluated based on training accuracy, testing accuracy, validation accuracy, and validation loss metrices. Moreover, a comparative analysis is done with Residual Neural Network (ResNet) and Convolutional Neural Network (CNN) using the same datasets. The findings from this study prove that RAN provides the best performance among the three algorithms. ResNet has good performance, with an accuracy ranging from $67 \%$ to $87 \%$. The standard CNN algorithm does not perform well, with a very inconsistent accuracy of between $57 \%$ and $71 \%$. RAN produces the highest and most consistent accuracy, which is $94 \%$ and above. Further explanation is provided in this paper to prove the efficiency of RAN for the classification of brain tumours.
\end{abstract}

Keywords - Brain tumour, classification, deep learning with Residual Attention Network, MRI dataset

\section{INTRODUCTION}

Brain tumour classification requires a very tedious process as it is extremely difficult to distinguish between the different types of brain tumours [1, 2]. Early detection of brain tumour is crucial for initiating the stages of treatment [3]. After clinical detection, MRI is used to locate the tumour and estimate its size and type. All these pieces of information are needed for various purposes-- producing an accurate diagnosis and prescribing an effective therapy such as surgery, radiation, or chemotherapy. Studies have proven that the earlier the tumour is detected, the higher are the chances of survival of the cancer-affected patients [4]. These studies show how crucial brain imaging and appropriate classification tools are to the medical fraternity. In a study conducted by Cheng et al. [5], three approaches are used to classify brain tumours - bag-of-words, grey level co-occurrence matrix (GLCM), and intensity histogram. However, these are highly specialised methods; the person who uses these methods must be well versed with the tumour. In today's rapidly changing world, an efficient and organised brain tumour classification algorithm remains a priority, which is needed to increase the medical industry's diagnosis accuracy; and at this juncture, machine learning and deep learning come to work as a good alternative.
Several algorithms proposed by researchers in their investigations are relevant to our study. In a research conducted by R.J Deshmukh and R.S Khule [6], a Neurofuzzy algorithm is proposed. This algorithm combines both fuzzy logic and artificial neural network to detect brain tumours. The neuro-fuzzy classifier has been built to detect the various kinds of brain tumours. The features obtained from the MRI images of cancer patients are processed using the Neuro-Fuzzy classifier.

In addition, another researcher combines the Probabilistic Neural Network (PNN) and Backpropagation network (BPN) for the classification of brain tumours [7]. A probabilistic Neural Network is suitable for classification purposes since it is a radial basis network. The MRI scans used in this research consist of patients with Astrocytoma type of brain tumours. The tumour region in the MRI scan is removed from the picture through the Histogram Equalisation and Threshold method (segmentation). BPN is used in the supervised learning. Neural networks are highly generalisable, and the features can be extracted with image input [8].

Moreover, a study conducted by Paul, et al. [9] proposes a convolutional neural network $(\mathrm{CNN})$ for brain tumour classification. Convolutional neural networks are known to perform well with datasets consisting of pictures that make a good choice of the MRI datasets of different brain tumours. Based on the results produced from the model, the accuracy calculated is $91.43 \%$. The per image accuracy is also consistent with the per-patient accuracy, which shows the consistency of prediction. The results produced by this research prove that training convolutional neural network improves brain tumour classification by a milestone. CNN is one of the deep learning methods. Deep learning is a field of machine learning that aims to learn high-level abstractions of data by utilising hierarchical architectures [10]. The flourishing growth of deep learning produces many methods to detect brain tumours. Over the years, many deep learning methods have been produced, like Back Propagation Neural Network [11], Probabilistic Neural Network [12], and Deep Neural Network [13-18]. However, the accuracy and efficiency of these methods can be further improved. Both the industry and academia are strongly encouraged to conduct additional research into deep learning models for brain tumour classification in this context. This developmental direction can significantly reduce the target deep learning model's overall running time, ultimately matching the innovative health care and clinical practice's practical constraints [19]. One avenue for 